\title{
The optical properties of galaxies in the Ophiuchus cluster ${ }^{\star}$
}

\author{
F. Durret ${ }^{1}$, K. Wakamatsu ${ }^{2}$, C. Adami ${ }^{3}$, T. Nagayama ${ }^{4}$, and J. M. Omega Muleka Mwewa Mwaba ${ }^{5,6}$ \\ ${ }^{1}$ Sorbonne Université, CNRS, UMR 7095, Institut d'Astrophysique de Paris, 98bis Bd Arago, 75014 Paris, France \\ e-mail: durret@iap.fr \\ 2 Faculty of Engineering, Gifu University, 1-1 Yanagido, Gifu 501-1193, Japan \\ ${ }^{3}$ LAM, OAMP, Pôle de l'Etoile Site Château-Gombert, 38 rue Frédéric Joliot-Curie, 13388 Marseille Cedex 13, France \\ ${ }^{4}$ Department of Astrophysics, Kagoshima University, Nagoya, Japan \\ ${ }^{5}$ Universidade Federal de Sergipe, Departamento de Física, Av. Marechal Rondon, S/N, 49000-000 São Cristóvão, SE, Brazil \\ ${ }^{6}$ CAPES Foundation, Ministry of Education of Brazil, Brasilia â DF, 70.040-020, Brazil
}

Received 14 June 2017 / Accepted 24 January 2018

\begin{abstract}
Context. Ophiuchus is one of the most massive clusters known, but due to its low Galactic latitude its optical properties remain poorly known.

Aims. We investigate the optical properties of Ophiuchus to obtain clues on the formation epoch of this cluster, and compare them to those of the Coma cluster, which is comparable in mass to Ophiuchus but much more dynamically disturbed.

Methods. Based on a deep image of the Ophiuchus cluster in the $r^{\prime}$ band obtained at the Canada France Hawaii Telescope with the MegaCam camera, we have applied an iterative process to subtract the contribution of the numerous stars that, due to the low Galactic latitude of the cluster, pollute the image, and have obtained a photometric catalogue of 2818 galaxies fully complete at $r^{\prime}=20.5$ mag and still $91 \%$ complete at $r^{\prime}=21.5$ mag. We use this catalogue to derive the cluster Galaxy Luminosity Function (GLF) for the overall image and for a region (hereafter the "rectangle" region) covering exactly the same physical size as the region in which the GLF of the Coma cluster was previously studied. We then compute density maps based on an adaptive kernel technique, for different magnitude limits, and define three circular regions covering $0.08,0.08$, and $0.06 \mathrm{deg}^{2}$, respectively, centred on the cluster (C), on northwest (NW) of the cluster, and southeast (SE) of the cluster, in which we compute the GLFs.

Results. The GLF fits are much better when a Gaussian is added to the usual Schechter function, to account for the excess of very bright galaxies. Compared to Coma, Ophiuchus shows a strong excess of bright galaxies.

Conclusions. The properties of the two nearby very massive clusters Ophiuchus and Coma are quite comparable, though they seem embedded in different large-scale environments. Our interpretation is that Ophiuchus was built up long ago, as confirmed by its relaxed state (see paper I) while Coma is still in the process of forming.
\end{abstract}

Key words. galaxies: clusters: individual: Ophiuchus - galaxies: luminosity function, mass function - galaxies: photometry

\section{Introduction}

The Ophiuchus cluster is one of the most massive nearby clusters, at a redshift of $z=0.0296$. Since its discovery (Wakamatsu \& Malkan 1981; Johnston et al. 1981), it has been studied in detail in X-rays (see Werner et al. 2016 and references therein), but due to its low Galactic latitude $\left(b=9.3^{\circ}\right)$ its optical properties are not well known. In a previous paper (Durret et al. 2015, hereafter paper I), we have shown that overall the cluster can be considered as relaxed, composed of a main structure and a single, much smaller substructure, with a total mass of $1.1 \times 10^{15} M_{\odot}$. We present here our photometric catalogue of 2818 galaxies in the $r^{\prime}$ band, and discuss the galaxy luminosity function (GLF) in the overall image, in a region (hereafter the "rectangle" region) covering exactly the same physical size as the region in which the GLF of the Coma cluster was studied by Adami et al. (2007), and in three regions of the cluster.

In spite of numerous studies, GLFs do not seem to have properties that can be predicted based on the simple knowledge of the cluster mass or structure (relaxed or not). GLFs are usually

\footnotetext{
* The photometric catalogue of Ophiuchus (full Table B.1)

is only available at the CDS via anonymous ftp to cdsarc.u-strasbg.fr (130.79.128.5) or via

http://cdsarc.u-strasbg.fr/viz-bin/qcat?J/A+A/613/A20
}

fit by a Schechter function (Press \& Schechter 1974) but deviations from this function are often observed, in particular in merging clusters, which often show an excess of very bright galaxies. At the other end of the GLF, the faint end slope $\alpha$ provides information on the cluster formation and evolution (see e.g. Martinet et al. 2015). However, we note that $\alpha$ seems to vary from one cluster to another with no obvious dependence on the cluster properties. Deriving the GLF of Ophiuchus and comparing it to that of other massive nearby clusters such as Coma, which shows a much higher level of substructuring, could reveal interesting differences or similarities between two clusters of comparable mass, one relaxed and the other non-relaxed.

In a preliminary study based on an extensive redshift catalogue combining their own data with archive data from $6 \mathrm{dF}$ and NED, Wakamatsu et al. (2005) analysed the distribution of 4717 galaxies with recession velocities in the range $500<c z<40000 \mathrm{~km} \mathrm{~s}^{-1}$. They showed that Ophiuchus is sufficiently large and rich to be considered a supercluster, which they call the Ophiuchus supercluster. Wakamatsu et al. (2005) also detected a wall structure $65 \mathrm{Mpc}$ in length between Ophiuchus and a zone from where the Hercules supercluster extends to the north. They noted that this wall runs close to northsouth and crosses the Great Wall perpendicularly at the Hercules supercluster. Another wall could be a continuation of 
the Ophiuchus-Hercules wall across and beyond the Galactic plane. They also found a strong deficiency of galaxies with velocities $c z<4000 \mathrm{~km} \mathrm{~s}^{-1}$, thus extending the Local Void beyond the limit determined by Tully \& Fisher (1987). So Ophiuchus seems to show particularities at large scales, and this will be investigated in a companion paper (Wakamatsu et al. in prep.)

As in paper I, we adopt the following quantities: cluster centre $\mathrm{RA}=258.1155^{\circ}$, Dec $=-23.3698^{\circ}$ (coordinates of the $\mathrm{cD}$ galaxy), a scale of $0.585 \mathrm{kpc} / \mathrm{arcsec}$, and a distance modulus of 35.54 .

\section{The photometric galaxy catalogue}

The study presented here is based on the full exploitation of the CFHT/MegaCam image that we obtained in the $r^{\prime}$ band. Magnitudes have been measured in the AB photometric system. At the redshift of Ophiuchus, the $1 \times 1 \mathrm{deg}^{2}$ MegaCam field corresponds to a region of about $2.1 \times 2.1 \mathrm{Mpc}^{2}$. This covers part of the cluster (for which $r_{200}=2.1 \mathrm{Mpc}$ (see Table 2 in Paper I). As explained in Paper I for our pilot survey in a small area $\left(10 \times 9.5 \mathrm{arcmin}^{2}\right)$ centred on the cD galaxy, an automated galaxy survey did not work properly for the Ophiuchus cluster due to the high density of foreground stars. Instead, we made our galaxy survey based on the visual inspection of star-subtracted images, which are created from original ones with a PSF-deblending algorithm. After this process, galaxies are fairly easily found. To make the survey as uniform as possible for the present large sky area (about $1 \mathrm{deg}^{2}$ ), we set the limiting magnitude about 1.5 mag brighter than the previous pilot survey and iterated the survey four times, going to fainter magnitudes step by step. This work was done by one of us (K.W.) and his research assistant. Finally we picked 2818 galaxies, including 227 galaxies that were in the pilot survey of Paper I.

We now produce a full $r^{\prime}$ band magnitude catalogue for the entire $1 \mathrm{deg}^{2}$ region covered by MegaCam, including the galaxies from Paper I, and containing 2818 galaxies. Since our observations were made in 2010, the $r^{\prime}$ band filter mounted on MegaCam was a first generation filter. Magnitudes in this filter (in the $\mathrm{AB}$ system) are very close to the SDSS magnitudes: as indicated in the MegaCam pages ${ }^{1}$, the relation between the MegaCam and SDSS $r$ band magnitudes is:

$r_{\text {MegaCam }}-r_{\text {SDSS }}=-0.024\left(g_{\mathrm{SDSS}}-r_{\mathrm{SDSS}}\right)$.

For elliptical galaxies at redshift $z=0$ (the galaxy type expected to be dominant in Ophiuchus), Fukugita et al. (1995) give $g_{\text {SDSS }}-r_{\text {SDSS }}=0.77$, in which case $r_{\text {MegaCam }}$ $=r_{\mathrm{SDSS}}-0.013$. For other types of galaxies, the difference between $r_{\text {MegaCam }}$ and $r_{\text {SDSS }}$ will be even smaller. In view of the error bars on magnitudes (see Table A.1) and of the large extinction correction that must be applied (see below), we hereafter neglect the difference between $r_{\text {MegaCam }}$ and $r_{\text {SDSS }}$. All the galaxies were treated as described in Paper I (iterative star subtraction) and subimages were extracted around each galaxy after the stars were eliminated. The varying extinction in the field results in a different sensitivity to galaxy intrinsic surface brightness with position. This will impact photometric methods based on a thresholding in surface brightness for object measurements, such as isophotal magnitudes or Kron magnitudes. For this reason, we have used SExtractor MAG_MODEL magnitudes based on a profile fit rather than the classical MAG_AUTO. The PSF

1 http://www.cadc-ccda.hia-iha.nrc-cnrc.gc.ca/en/ megapipe/docs/filt.html

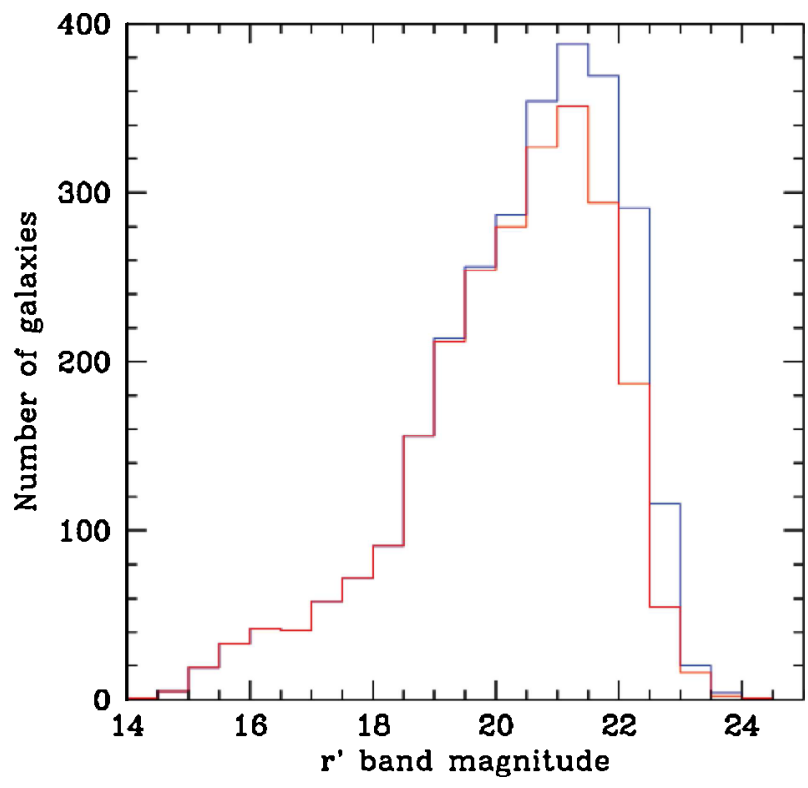

Fig. 1. Observed magnitude histogram in the $r^{\prime}$ band for the galaxies included in the $1 \mathrm{deg}^{2}$ region covered by our MegaCam image (before applying any extinction correction). The 2499 galaxies of the preliminary survey are shown in red and the 319 galaxies of our second survey are shown in blue.

was measured with PSFEx ${ }^{2}$, and a Sersic profile convolved with this PSF was fit to each galaxy using SExtractor (Bertin \& Arnouts 1996), giving MAG_MODEL magnitudes.

To illustrate the depth of the photometric catalogue, we show in Fig. 1 the corresponding apparent magnitude histogram. Nearly half of the galaxies in the three faintest bins in Fig. 1 are galaxies found in the pilot survey given in Paper I, implying that the present galaxy survey in the large area is shallower than the pilot survey.

To check the completeness of our preliminary galaxy survey containing 2499 galaxies, we marked on the star-subtracted images the detected galaxies with $20.0<r^{\prime}<22.0$ with different symbols in 0.5 mag intervals, and made the galaxy survey again for the full $1 \mathrm{deg}^{2}$ area. We did our best not to miss them during the survey process by changing the contrast and/or brightness level on the ds9 image viewer. Out of these newly detected galaxies $(\sim 450)$, photometric measurements were made for 319 galaxies among the brightest. The present catalog therefore comprises $2499+319=2818$ objects.

The magnitudes of the five brightest galaxies among these newly detected objects are 19.44, 19.85, 19.90, 20.13, and 20.33 mag, respectively. Also follow 12, 14, 13, and 24 galaxies, in the successive magnitude ranges from 20.5 to 21.5 with a step of $0.25 \mathrm{mag}$. One quarter of the 319 galaxies are brighter than $21.5 \mathrm{mag}$, while three quarters are fainter than this magnitude (Fig. 1). Based on these counts, we estimate that the catalogue is fully complete down to $20.5 \mathrm{mag}$, and almost complete $(98.5 \%)$ down to $20.75 \mathrm{mag}$. However, when the sensitivity variations among the 25 CCD detectors of MegaCam as well as the existence of faint diffuse nebular emission in this area of the Galactic plane are taken into account, there may still exist missed galaxies brighter than $20.5 \mathrm{mag}$, for example, diffuse galaxies of low surface brightness or compact galaxies of small ( 2.5 arcsec) angular diameter. The former may be missed due to irregularities of the sky background, while the latter can be hidden by the

2 http://www.astromatic.net/ 


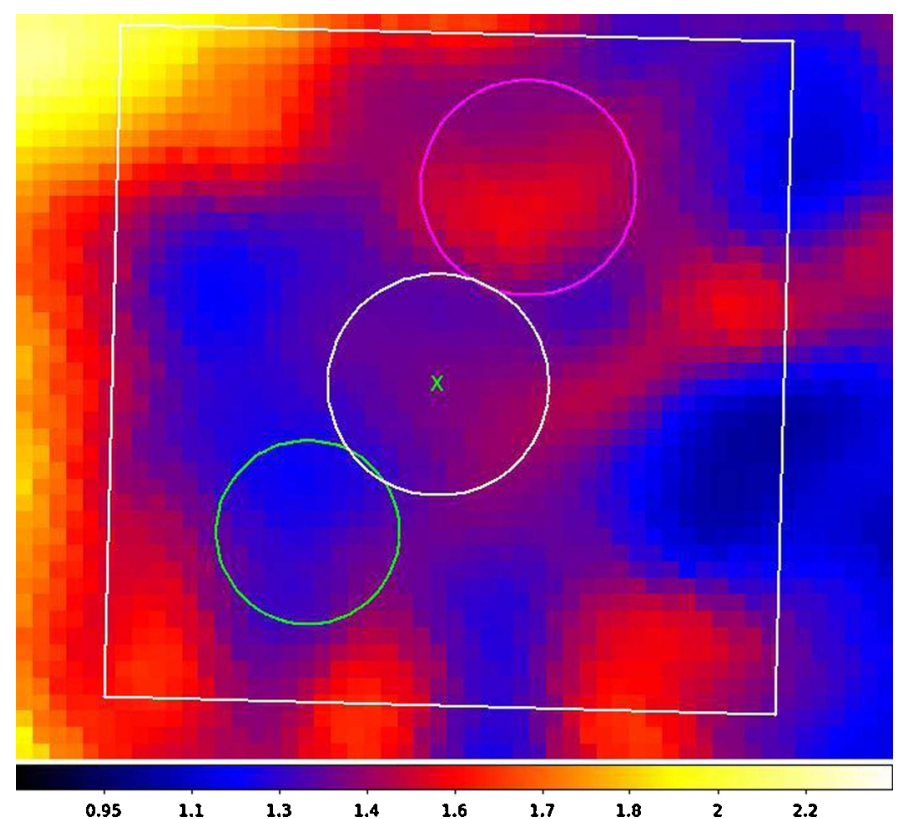

Fig. 2. Galactic extinction map in the Ophiuchus region. The white square shows the MegaCam field, and the three circles are those defined in Sect. 3.3. Extinction increases from blue to red and to yellow. The MegaCam field is oriented with North to the top and east to the left, and the extinction map is slightly tilted.

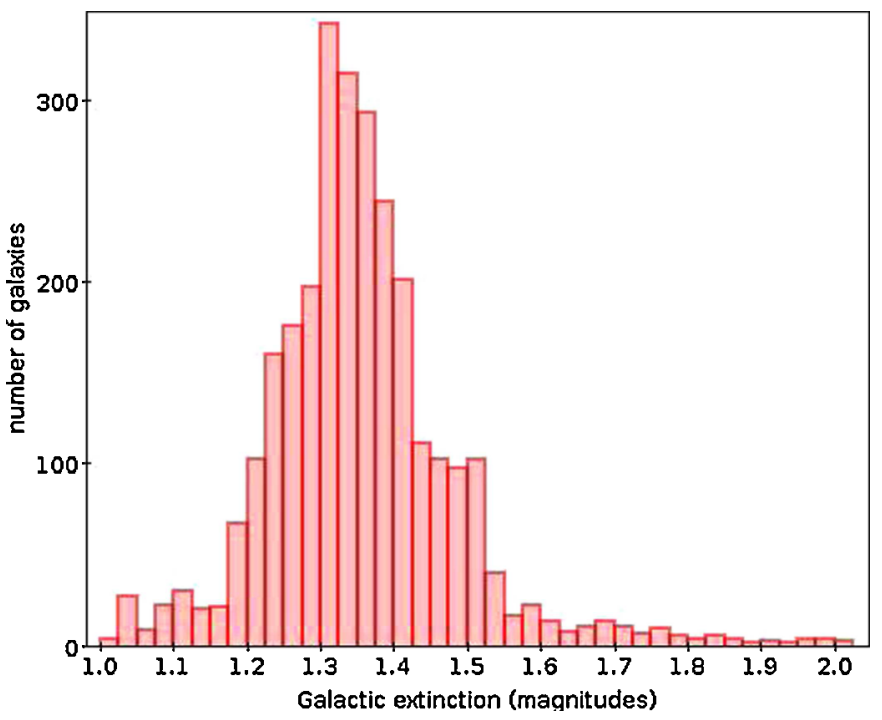

Fig. 3. Histogram of the Galactic extinction in the $r^{\prime}$ band for the 2818 galaxies of our photometric catalogue.

spiders of bright foreground stars or by multiply blended stars. We finally estimate that the present catalogue is really complete down to $r^{\prime}=20.25 \pm 0.25$ mag. The completeness in absolute magnitude is discussed in Sect. 3.1

We note that the shortage of galaxies fainter than $M_{r^{\prime}}=-20$ in zone $\mathrm{C}$ is real (see Sect. 3.4), and is not due to the incompleteness of our survey in the core region.

At such a low Galactic latitude, Galactic extinction is obviously large. We first adopted the value of $1.357 \mathrm{mag}$ given by NED for the SDSS $r$ band. We then decided to go one step further and look in more detail at the Galactic dust extinction map by Schlegel et al. (1998) with newer estimates from
Schlafly \& Finkbeiner $(2011)^{3}$. The map contains the values of the colour excess $E(B-V)$ with a pixel size of 1.5 arcmin. We converted it to an extinction map $A_{r}$ in the $r^{\prime}$ band, by multiplying it by $R=2.285$ (value taken from Schlafly \& Finkbeiner 2011, Table 6), that is, $A_{r}=R \times E(B-V)$.

The extinction varies quite strongly in the region covered by our image: between 1.025 and 2.02, as illustrated in Fig. 2. When computing GLFs in Sect. 3 we therefore applied either the constant extinction of $1.357 \mathrm{mag}$ or the individual extinction for each galaxy.

The heavy galactic extinction for the Ophiuchus cluster causes a reduction of the apparent angular size of each galaxy, which could require further extinction correction. However, this correction amounts to $0.15 \mathrm{mag}$ at most, and it depends in a complicated way on the adopted colour band and morphological type of each galaxy (Cameron 1990), so we decided not to apply it.

The final photometric catalogue of 2818 galaxies will be made available in ViZieR at the $\mathrm{CDS}^{4}$. For each galaxy, it contains the following information: RA, Dec, measured $r^{\prime}$ band magnitude with no extinction correction, magnitude corrected for a constant value of $1.357 \mathrm{mag}$, magnitude corrected for the individual extinction of each galaxy, major and minor axes $(a$ and $b$ ), position angle (PA) of the major axis, and error on the PA. The values of $a$ and $b$ are the angular sizes of the semi-major and -minor axes which are computed from the $1 \sigma$ values of the half-axes given by SExtractor, multiplied by a factor 4.0 to fully enclose the galaxies (as advised in the SExtractor manual). The typical errors on the magnitudes, estimated from a subsample of more than 40 galaxies measured twice (in adjacent fields), are given in Table A.1. The first ten lines of the catalogue are shown in Appendix B.

\section{The galaxy luminosity function of the Ophiuchus cluster}

\subsection{The global galaxy luminosity function}

To compute the GLF of the Ophiuchus cluster, it is necessary to statistically subtract the background contribution. Our original galaxy counts in the CFHT/MegaCam field are shown in Fig. 1. We first corrected them for a constant extinction of 1.357. Background counts were taken from Yasuda et al. (2001), who estimated field galaxy counts from the SDSS at relatively bright magnitudes. They show galaxy counts in the $r^{\prime}$ magnitude in their Fig. 4. Since these counts are taken in the same filter as our data, in bins of $0.5 \mathrm{mag}$, and normalized to $1 \mathrm{deg}^{2}$, we can subtract them from our counts in a straightforward way to obtain the GLF (after normalizing our data to the area covered by our image: $0.9882 \mathrm{deg}^{2}$ ). As a check to the Yasuda et al. counts, we also plotted on the same figure the VVDS counts by McCracken et al. (2003), that we cannot use here since they start at a magnitude of 18. We can see in Fig. 4 that the two sets of background counts match well. We converted apparent to absolute magnitudes by applying the distance modulus of 35.54, and thus obtained Fig. 4. In view of the small redshift of the cluster, we applied no $k$-correction.

However, we can see in Fig. 4 that the Yasuda background counts become larger than our galaxy counts for $M_{r^{\prime}} \sim-16.3$, corresponding to $r^{\prime} \sim 19.2$, a value which is more than one magnitude brighter than our completeness limit $\left(r^{\prime}=20.25\right)$. This leads us to think that due to cosmic variance the Yasuda

\footnotetext{
3 http://irsa.ipac.caltech.edu/applications/DUST/

4 http://vizier.u-strasbg.fr/viz-bin/VizieR
} 


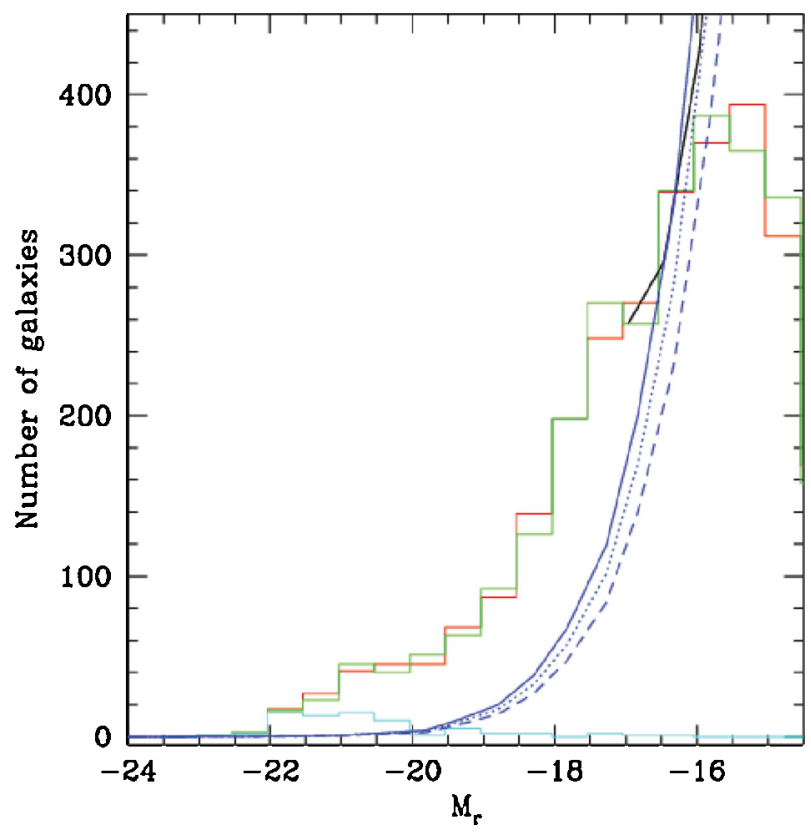

Fig. 4. Galaxy counts in the $r^{\prime}$ band converted to absolute magnitude (see text). The red histogram shows the 2818 galaxies from our catalogue and the cyan histogram those with spectroscopic redshifts in the cluster range, both corrected for a constant galactic extinction of 1.357 as explained in Sect. 2. The green histogram shows the 2818 galaxies from our catalogue corrected for their individual galactic extinction. The blue and black lines show the field galaxy counts computed by Yasuda et al. (2001), and McCracken et al. (2003) respectively. The blue dotted and dashed lines show the Yasuda background counts multiplied by 0.85 and 0.70 , respectively (see Sect. 3.1).
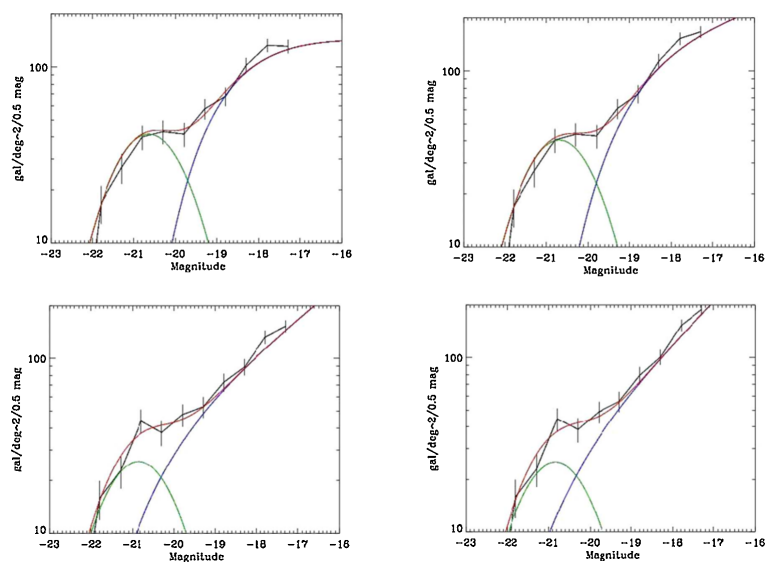

Fig. 5. Galaxy luminosity function of the Ophiuchus cluster in the entire MegaCam field. The black line and points are the data, the green curve is the Gaussian component, the blue curve is the Schechter function, and the red curve is the total of the two components. Top: constant galactic extinction correction of 1.357, bottom: individual galactic extinction correction. Left: subtraction of Yasuda background counts, and right: subtraction of Yasuda background counts multiplied by $f=0.7$ (see Sect. 3.1).

counts are an overestimate of the background counts in the direction of Ophiuchus. Our extensive redshift catalogue in this area (Wakamatsu et al. in prep., see also Wakamatsu et al. 2005) indeed shows that besides the foreground $\left(\mathrm{cz}<6000 \mathrm{~km} \mathrm{~s}^{-1}\right)$ local void, there is also a large background void behind the Ophiuchus cluster, up to $\mathrm{cz} \sim 16000 \mathrm{~km} \mathrm{~s}^{-1}$. Therefore the background contamination for the cluster should mostly come from the background galaxies with velocities $\mathrm{cz}>16000 \mathrm{~km} \mathrm{~s}^{-1}$.
To examine how Yasuda's background data are modified by this cosmic variance, we carried out numerical simulations by changing the values of various parameters of the Schechter function, such as $M^{*}, \alpha$, and the faintest magnitude cutoff, as well as the integration limit of the depth (distance modulus $(m-M)<40.5)$. The results show that these voids affect effectively the GLF parameters for $r^{\prime}>17$, or $M_{r^{\prime}}>-17.5$ in absolute magnitude. However, we cannot obtain a unique solution, and all we can say is that the Yasuda background counts should be multiplied by a factor $f=0.85 \pm 0.15$. We therefore fit the various GLFs with the values of $f$ that bracket this interval: $f=1.0$ and $f=0.70$, noted, respectively, $f$ and $f^{\prime}$ hereafter.

Since our galaxy counts show an excess of bright galaxies, we accounted for this excess with a Gaussian function $G(M)$ (the values of the reduced $\chi^{2}$ are indeed much lower when a Gaussian is included), and we fit the GLFs with a global function:

$G L F(M)=S(M)+G(M)$.

Here $S(M)$ is the Schechter function defined in terms of the absolute magnitude $M$ as:

$S(M)=0.4 \ln 10 \Phi^{*} y^{\alpha+1} e^{-y}$

with $y=10^{0.4\left(M^{*}-M\right)}$, where $\Phi^{*}$ is a normalisation factor, $M^{*}$ is the typical magnitude separating the bright and faint regimes of the GLF, and $\alpha$ is the faint end slope. $G(M)$ is a Gaussian function to account for the bright part:

$G(M)=A \exp \left[\left(-4 * \ln (2) *\left(M-M_{c}\right)^{2}\right) /\left(f w h m^{2}\right)\right]$

where $M_{c}$ is the central magnitude, fwhm is the full-width-athalf maximum and $A$ is the amplitude for $M=M_{c}$.

We also tried to fit the GLFs with a global function including the factor $f \leq 1$ by which the Yasuda background counts should be multiplied:

$G L F(M)=S(M)+G(M)+f * B(M)$.

However, the addition of a seventh free parameter made the solution quite uncertain: the reduced $\chi^{2}$ remained almost the same for all the values of $f$, and the $f$ parameter space did not seem to be explored properly, so it was not possible to estimate $f$ with this method. We therefore decided to fit all the GLFs with the sum of a Gaussian and a Schechter functions, multiplying the Yasuda counts by $f$ and $f^{\prime}$.

It should be noted that the large excess of bright galaxies in the GLFs is not affected by the ambiguity of the field galaxy correction, which is very small for bright galaxies.

We computed the GLF both for a constant (noted "ct." in Table 1) galactic extinction of 1.357 and for a galactic extinction that is different for each galaxy (noted "var." in Table 1), as explained above. The results of the Gauss+Schechter fits are shown in Fig. 5 and the corresponding parameters are given in Table 1 . The reduced $\chi^{2}$ values of the fits are 0.81 and 0.72 for a constant and a variable extinction correction, respectively. The reduced $\chi^{2}$ values are larger than 2.0 if no Gaussian component is included, therefore justifying our choice of a Gauss+Schechter fit to the GLFs.

We can see that the galaxy counts do not vary strongly with the method used to correct for galactic extinction (Fig. 4), as confirmed by the fact that the shapes and parameters of the GLF fits are quite similar in both cases, and agree within the error bars (Table 1). Of course if we take $f^{\prime}=0.7$ the counts are higher, and the parameters of the fit change. 
Table 1. Parameters of the GLF for the overall image, with magnitudes corrected for extinction with a constant value (ct.), or with their individual values (var.), as well as for the rectangular region with the same physical size as the GLF computed for the Coma cluster, and for the three circular regions $\mathrm{C}$, NW and SE (see text).

\begin{tabular}{lccccccc}
\hline \hline & $f$ & $\Phi^{*}$ & $M^{*}$ & $\alpha$ & $A$ & $M_{c}$ & $f w h m$ \\
\hline Overall (ct.) & 1.0 & $179 \pm 130$ & $-18.9 \pm 1.0$ & $-0.97 \pm 0.40$ & $42 \pm 7$ & $-20.6 \pm 0.3$ & $0.7 \pm 0.1$ \\
Overall (var.) & 1.0 & $145 \pm 137$ & $-19.2 \pm 1.1$ & $-1.19 \pm 0.36$ & $40 \pm 9$ & $-20.7 \pm 0.3$ & $0.7 \pm 0.1$ \\
Overall (ct.) & 0.7 & $34 \pm 115$ & $-20.8 \pm 3.8$ & $-1.48 \pm 0.37$ & $26 \pm 34$ & $-20.9 \pm 0.3$ & $0.7 \pm 0.2$ \\
Overall (var.) & 0.7 & $25 \pm 52$ & $-21.1 \pm 2.6$ & $-1.59 \pm 0.19$ & $25 \pm 19$ & $-20.8 \pm 0.2$ & $0.7 \pm 0.2$ \\
\hline Rectangle (ct.) & 1.0 & $317 \pm 108$ & $-18.7 \pm 0.8$ & $-0.76 \pm 0.28$ & $65 \pm 10$ & $-20.5 \pm 2.2$ & $1.1 \pm 0.5$ \\
Rectangle (var.) & 1.0 & $177 \pm 120$ & $-19.7 \pm 1.0$ & $-1.08 \pm 0.25$ & $56 \pm 18$ & $-20.8 \pm 0.3$ & $0.7 \pm 0.1$ \\
Rectangle (ct.) & 0.7 & $217 \pm 268$ & $-19.4 \pm 2.0$ & $-1.07 \pm 0.51$ & $58 \pm 25$ & $-20.9 \pm 0.8$ & $0.9 \pm 0.5$ \\
Rectangle (var.) & 0.7 & $90 \pm 78$ & $-20.6 \pm 1.3$ & $-1.32 \pm 0.16$ & $41 \pm 25$ & $-20.9 \pm 0.2$ & $0.6 \pm 0.1$ \\
\hline C (ct.) & 1.0 & $428 \pm 127$ & $-19.4 \pm 0.4$ & $-0.81 \pm 0.22$ & $80 \pm 4$ & $-21.3 \pm 0.1$ & $0.7 \pm 0.2$ \\
C (var.) & 1.0 & $551 \pm 112$ & $-18.8 \pm 0.4$ & $-0.58 \pm 0.23$ & $76 \pm 6$ & $-21.0 \pm 0.4$ & $1.4 \pm 1.0$ \\
C (ct.) & 0.7 & $435 \pm 41$ & $-19.4 \pm 0.2$ & $-0.83 \pm 0.04$ & $86 \pm 8$ & $-21.3 \pm 0.1$ & $0.6 \pm 0.1$ \\
C (var.) & 0.7 & $437 \pm 49$ & $-19.4 \pm 0.2$ & $-0.83 \pm 0.05$ & $76 \pm 7$ & $-21.3 \pm 0.2$ & $0.8 \pm 0.2$ \\
\hline NW (ct.) & 1.0 & $2 \pm 4$ & $-34.5 \pm 7.9$ & $-1.28 \pm 0.04$ & & & \\
NW (var.) & 1.0 & $4 \pm 6$ & $-31.4 \pm 5.3$ & $-1.28 \pm 0.02$ & & & \\
NW (ct.) & 0.7 & $82 \pm 54$ & $-19.8 \pm 0.8$ & $-1.35 \pm 0.15$ & $81 \pm 10$ & $-20.8 \pm 0.1$ & $0.6 \pm 0.1$ \\
NW (var.) & 0.7 & $379 \pm 62$ & $-17.5 \pm 0.3$ & $-0.47 \pm 0.24$ & $81 \pm 6$ & $-20.2 \pm 0.2$ & $1.5 \pm 0.3$ \\
\hline SE (ct.) & 1.0 & $16 \pm 14$ & $-22.9 \pm 1.3$ & $-1.51 \pm 0.07$ & & & \\
SE (var.) & 1.0 & $0.6 \pm 1.0$ & $-31.9 \pm 4.4$ & $-1.43 \pm 0.02$ & & & \\
SE (ct.) & 0.7 & $537 \pm 57$ & $-17.4 \pm 0.2$ & $0.42 \pm 0.34$ & $74 \pm 6$ & $-20.3 \pm 0.1$ & $0.8 \pm 0.1$ \\
SE (var.) & 0.7 & $573 \pm 63$ & $-17.8 \pm 0.3$ & $-0.31 \pm 0.31$ & $91 \pm 7$ & $-20.3 \pm 0.1$ & $0.6 \pm 0.1$ \\
\hline
\end{tabular}

Notes. $f$ is the factor by which the Yasuda background counts were multiplied to account for cosmic variance.

We note that the faintest bins in which we can compute the GLF are at absolute magnitudes of -17.5 and -16.5 , for $f=1.0$ and $f^{\prime}=0.7$, respectively, brighter or comparable to the completeness limit of our photometric catalogue $(-16.4)$ given in the previous section; therefore they can be considered as reliable.

The fits appear quite satisfactory in view of all the difficulties overcome to obtain a photometric catalogue. The Gaussian components are in both cases important at bright magnitudes. An excess at very bright magnitudes seems to be quite a general phenomenon, as already noted for example in Abell 223 (Durret et al. 2010), Abell 1758 (Durret et al. 2011) or Abell 3376 (Durret et al. 2013). But all these clusters were mergers, so we did not expect to find such a large Gaussian component in Ophiuchus, which is believed from previous studies to be quite relaxed. The faint end slope is moderate for $f=1.0(\alpha=-0.97$ or -1.19 , depending on the way the extinction is corrected), and steeper for $f^{\prime}=0.7(-1.48$ to -1.59$)$.

The latter steep values could be due to a non-negligible background contamination at faint magnitudes. However we note that comparable values have already been observed in other clusters. For example, in the nearby massive cluster Coma, Adami et al. (2007) found a faint end slope even steeper than -1.49 in the north part of Coma, which is a relatively quiescent region, and a flatter slope around -1.3 in the south half of Coma, which is experiencing infalls.

This led us to derive the GLF in a region having the same physical size as for Coma, to make the comparison of these two clusters more reliable. Our results are described in the following section.

\subsection{The galaxy luminosity function in a physical region similar to our previous study of the Coma GLF}

Adami et al. (2007) analysed the GLF of the Coma cluster in a region covering 40 arcmin in right ascension and 50 arcmin
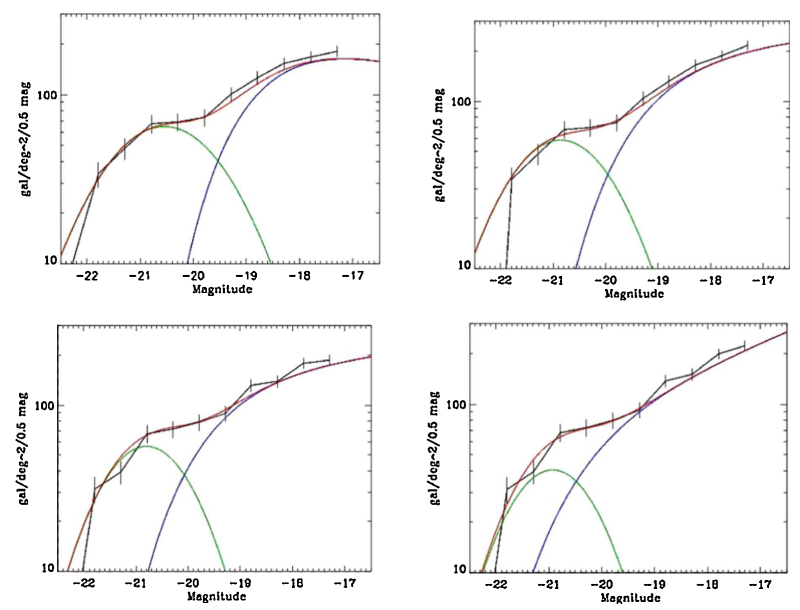

Fig. 6. Galaxy luminosity function of the Ophiuchus cluster in the "rectangle" region (covering the same physical size as for the Coma cluster; see text). Top: constant galactic extinction, bottom: individual galactic extinction correction. Left: subtraction of Yasuda background counts, and right: subtraction of Yasuda background counts multiplied by $f=0.7$ (see Sect. 3.1). See caption of Fig. 5 for colour coordination of lines and curves.

in declination. At the redshift of Coma, this corresponds to a zone of $1.1136 \times 1.3920 \mathrm{Mpc}^{2}$. Since the scale for Ophiuchus is $0.585 \mathrm{kpc} \operatorname{arcsec}^{-1}$, this size corresponds to a region of $0.5288 \times 0.661 \mathrm{deg}^{2}$, or a surface of $0.34954 \mathrm{deg}^{2}$. We therefore extracted from our photometric catalogue a zone defined by $257.8511<\mathrm{RA}<258.3799,-23.7003<$ Dec $<-23.0393$, containing 1248 galaxies.

We computed the GLF in the same way as described above and fit a Gaussian and a Schechter functions. The corresponding GLFs and their fits are shown in Fig. 6 for two cases of different 
absorption corrections and the best fit parameters are given in Table 1 (region noted "rectangle").

We compare these GLFs with that obtained for Coma by Adami et al. (2007) in Sect. 4.

\subsection{Defining regions in Ophiuchus through density maps}

GLFs have been observed to vary with the position within the cluster (e.g. Adami et al. 2007 and references therein). In order to define the regions where it would be interesting to derive GLFs, we computed galaxy density maps based on an adaptive kernel technique with a generalized Epanechnikov kernel as suggested by Silverman (1986). Our method is based on an earlier version developed by Timothy Beers (ADAPT2) and further improved by Biviano et al. (1996). The statistical significance is established by bootstrap resampling of the data. A density map is computed for each new realisation of the distribution. We choose a pixel size of $0.001^{\circ}(3.6 \mathrm{arcsec})$. For each pixel of the map, the final value is taken as the mean over all realisations. A mean bootstrapped map of the distribution is thus obtained. The number of bootstraps used here is 100 .

The significance level of our detections was estimated from the mean value and dispersion of the background of each image. To estimate these quantities, we draw for each density map the histogram of the pixel intensities. We apply a $2.5 \sigma$ clipping to eliminate the pixels of the image that have high values and correspond to objects in the image. We then redraw the histogram of the pixel intensities after clipping and fit this distribution with a Gaussian. The mean value of the Gaussian gives the mean background level, and the width of the Gaussian gives the dispersion, that we call $\sigma$. We then compute the values of the contours corresponding to $3 \sigma$ detections as the background plus $3 \sigma$. The contours shown in Fig. 7 start at $3 \sigma$ and increase by $1 \sigma$.

The limitation here is that we only have photometry in one band, so we cannot select galaxies along the red sequence (i.e. with a high probability of belonging to the cluster). We therefore chose to compute maps for different magnitude limits (chosen arbitrarily), applied to the magnitudes before any extinction correction: $r^{\prime}<15.5$ (25 galaxies), $r^{\prime}<16$ (58 galaxies), $r^{\prime}<17$ (141 galaxies), $r^{\prime}<18$ (271 galaxies), and $r^{\prime}<19$ (517 galaxies). Obviously, the contamination by background galaxies becomes larger as the limiting cut becomes fainter.

The resulting density maps are shown in Fig. 7. Since the galaxy catalogues from which the density maps are computed have slightly different sizes, the maps also have somewhat different sizes. However, the radii of the three circles remain constant from one figure to another.

We can see that depending on the magnitude cut, the aspects of the density maps change. The cluster (white circle) is clearly the brightest structure in all the maps. However, a structure is visible northwest of the cluster, and a second structure appears at fainter magnitudes southeast of the cluster.

We therefore defined three circular regions in which we derived the GLFs: the first one (hereafter C) is centred on the cluster centre and has a radius of 10.0 arcmin, or $351 \mathrm{kpc}$ at the cluster redshift (white circle), the northwest circle (hereafter $\mathrm{NW})$ is centred on RA $=257.9774^{\circ}$, Dec $=-23.0701^{\circ}$ and has a radius of 9.7 arcmin, or $340 \mathrm{kpc}$ (magenta circle), and the southeast circle (hereafter SE) is centred on RA $=258.3224^{\circ}$, Dec $=-23.5961^{\circ}$ and has a radius of $8.3 \mathrm{arcmin}$, or $291 \mathrm{kpc}$ (green circle).

We note that the NW region more or less coincides with the zone where Watanabe et al. (2001) found an excess of X-ray
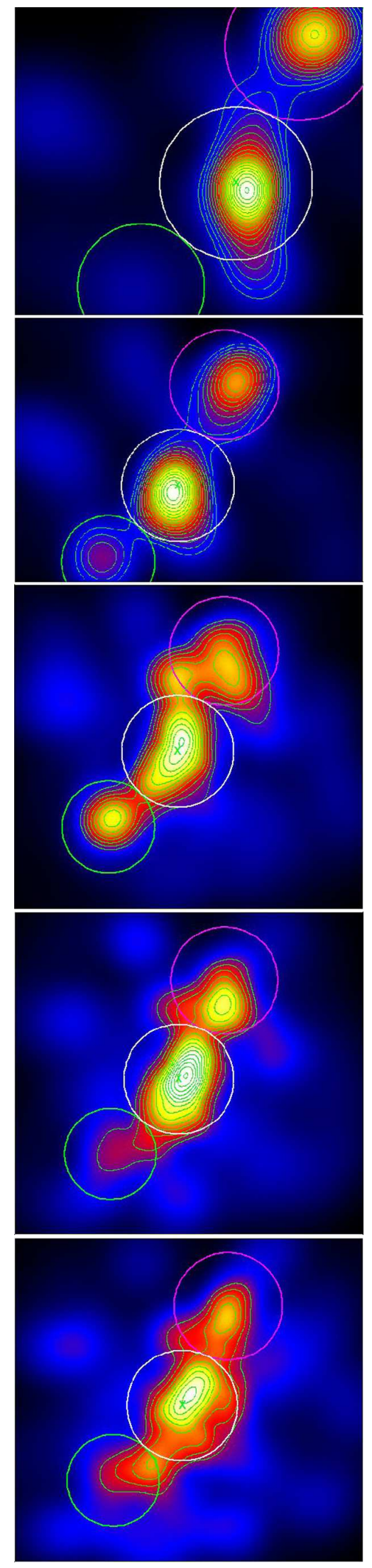

Fig. 7. Galaxy density maps with various magnitude limits (chosen arbitrarily, for magnitudes without any extinction correction). From top to bottom: $r<15.5$ (25 galaxies), $r<16$ (58 galaxies), $r<17$ (141 galaxies), $r<18$ (271 galaxies), and $r<19$ (517 galaxies). Contour levels start at $3 \sigma$ above the background (see text) and are spaced by $1 \sigma$. The cross indicates the position of the cD galaxy. 

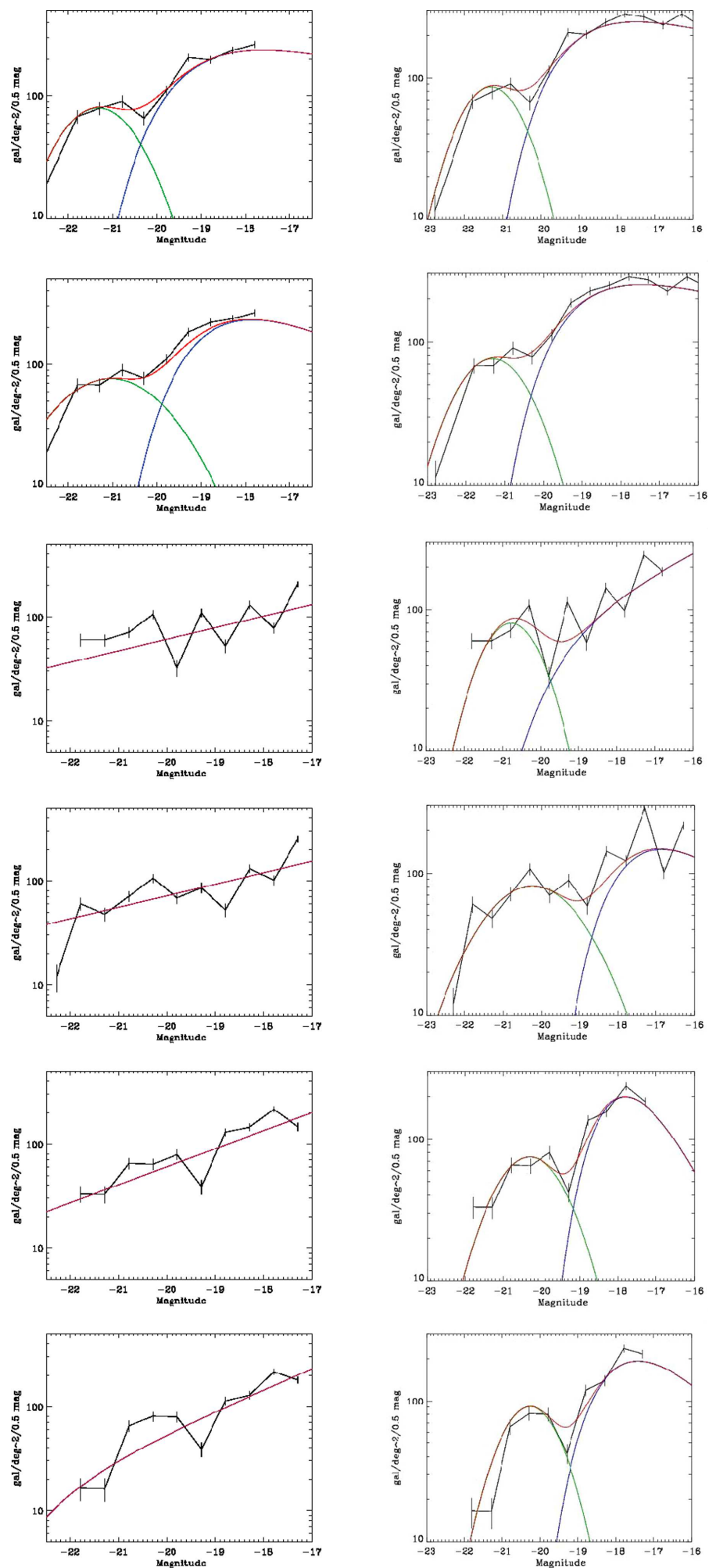

Fig. 8. Galaxy luminosity functions in the Centre $\mathrm{C}$ (two top figures), and in regions NW (two middle figures) and SE (two bottom figures) (see text). For each region, the top figure was obtained with a constant Galactic extinction and the bottom one with a different extinction correction for each galaxy. Left: subtraction of Yasuda background counts, and right: subtraction of Yasuda background counts multiplied by $f=0.7$ (see Sect. 3.1). The line colours are as in Fig. 5 .

emission in their ASCA image (see their Fig. 5), so it is probably a substructure in the cluster.

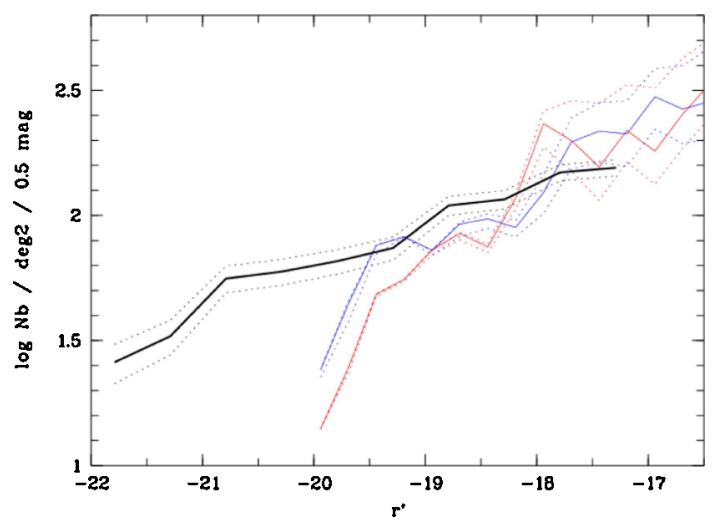

Fig. 9. Comparison of the GLFs of Ophiuchus (computed with variable extinction and $f=1.0$ ) and Coma in regions with the same physical sizes. The Ophiuchus GLF is in black, while the GLFs of the North and South zones of Coma are in red and blue, respectively. The dotted lines show the $\pm 1 \sigma$ errors on each curve.

\subsection{The galaxy luminosity function in three regions}

We extracted galaxy counts in the three circular regions, $\mathrm{C}$, NW, and SE, and applied to each the two extinction corrections described above, and the two possible values for the background subtraction $\left(f=1.0\right.$ and $\left.f^{\prime}=0.7\right)$.

The surfaces covered by the regions $\mathrm{C}, \mathrm{NW}$, and SE are $0.087468,0.082725$, and $0.060056 \mathrm{deg}^{2}$, respectively. The GLFs are plotted in Fig. 8 and the fit parameters are given in Table 1.

In region $\mathrm{C}$, which corresponds to the cluster core, the importance of the Gaussian component is strong, as it was for the overall cluster (see Sect. 3.1) and in the "rectangle" region. The faint end slope is somewhat flatter in region $\mathrm{C}$ than in the overall cluster. Region $\mathrm{C}$ is therefore dominated by bright galaxies, as for region "rectangle".

In view of the shapes and poor qualities of the GLFs in regions $\mathrm{NW}$ and $\mathrm{SE}$, due to the low numbers of galaxies in these regions, no Gaussian function needs to be included for $f=1.0$. In fact a simple power law would be sufficient to fit the GLFs, since in most cases $M^{*}$ is not constrained. This implies that very bright galaxies are concentrated in the inner zones of the cluster, as expected, and that there are very few bright galaxies in the outer regions. The faint end slopes of the GLFs in regions NW and SE are close to those found for the overall image. We also note the importance of the Galactic extinction correction that somewhat modifies the parameters of the GLFs, in particular in the SE zone, where the extinction has a larger value (see Fig. 2). For $f^{\prime}=0.7$, the GLFs in regions NW and SE look much better, as expected, and the Gaussian component again appears necessary to account for the excess of bright galaxies.

\section{Discussion and conclusions}

Due to its low Galactic latitude, the Ophiuchus cluster is difficult to study at optical wavelengths. However, based on a photometric catalogue of 2818 galaxies in a zone covering $1 \mathrm{deg}^{2}$ around Ophiuchus, we have succeeded in obtaining the GLFs in the overall image, in a region (the "rectangle" region) of the same physical size as that considered for the Coma cluster by Adami et al. (2007), as well as in three circular regions defined from density maps: a central zone (C), and two regions northwest (NW) and southeast (SE) of the centre. In all the regions, the GLFs show an excess of very bright galaxies over a Schechter 
function that is well fit by a Gaussian. The faint end slope is quite flat in the very central region $\mathrm{C}$ : between -0.58 and -0.83 , while it is steeper in the outer zones. However, we must note that the GLFs of zones NW and SE are rather noisy, and that the choice of the correction for Galactic extinction and of the factor by which to multiply the background counts modifies the best fit parameters, so it is difficult to draw conclusions for these zones.

Since Ophiuchus and Coma are two massive nearby clusters, it is quite logical to compare their properties. The former is known to be quite relaxed (see e.g. Paper I) while extensive studies of the latter imply that it is far from relaxed and still undergoing one or several mergers. Therefore, the comparison of these two clusters can provide information on the effect the environment may have on their properties.

As explained above, we have extracted the Ophiuchus GLF in a region covering the same physical size (roughly $1.1 \times 1.4 \mathrm{Mpc}^{2}$ ) as the region in which the Coma GLF was calculated by Adami et al. (2007). The GLFs of Ophiuchus (computed for $f=1.0$ ) and of the North and South halves of Coma computed in regions of identical physical size are shown in Fig. 9. We can see from this figure that the GLFs are quite similar for galaxies fainter than $M_{r^{\prime}} \sim-19.5$, but that Ophiuchus shows a large excess, with respect to Coma, of galaxies brighter than $M_{r^{\prime}} \sim-19.5$. The North region of Coma is known to contain a large fraction of faint galaxies, while the South region of Coma, which is in contact with a large-scale filament arriving from the South-West, contains more bright galaxies. Ophiuchus contains many more bright galaxies than the South region of Coma, thus requiring mergers of quite massive galaxies.

One explanation could be that in the core of Ophiuchus many galaxy mergers take place. This agrees with the flatter faint end slope of the GLF in region C, relative to the two adjacent regions (NW and SE), and relative to the cluster at a larger scale (in particular the Overall region, see Table 1).

Mamon (1992) showed that for a galaxy of mass $m$, the merging rate in a cluster varies as $m^{2} / \sigma_{v}^{3}$, where $\sigma_{v}$ is the cluster velocity dispersion. The respective velocity dispersions of Ophiuchus and Coma are approximately $950 \mathrm{~km} \mathrm{~s}^{-1}$ (Paper I) and $1200 \mathrm{~km} \mathrm{~s}^{-1}$ (Adami et al. 2009), so the ratio of the merging rates in Ophiuchus and Coma for a galaxy of given mass $m$ is expected to be $\sim(1200 / 950)^{3}=2.0$. At the absolute magnitude $M_{r}=-20$, there are roughly three times as many galaxies in Ophiuchus as in Coma (Fig. 9). To obtain this factor of three, the ratio of the masses of bright galaxies in Ophiuchus and in Coma would therefore need to obey the relation $\left(m_{\text {Oph }} / m_{\text {Coma }}\right)^{2} \sim 3 / 2$, corresponding to bright galaxy masses in Ophiuchus $m_{\mathrm{Oph}}$ larger than the galaxy masses in Coma $m_{\text {Coma }}$ by about $20-25 \%$; values which are plausible. This very simple order of magnitude calculation shows that numerous galaxy mergers in the centre of Ophiuchus can indeed probably account for the very high number of galaxies brighter than $\sim-19.5$.

Another explanation to the high number of mergers could be that because Ophiuchus is embedded in a large-scale system involving the Great Attractor, this could favour galaxygalaxy interactions and mergers. Such mergers could involve bright field galaxies of comparable masses and/or galaxies with small relative velocities, favouring efficient energy exchange between these galaxies, and forming even brighter merged objects.
We note however that, though being a very rich and massive cluster, Ophiuchus does not appear to be embedded in a very dense network of galaxies, and its environment does not appear to be dense (Wakamatsu et al. in prep.). On the other hand, the large-scale environment of Coma, which is also a very rich cluster, at a comparable redshift, shows a much higher galaxy density. In the current paradigm where clusters are at the intersection of cosmic web nodes, it is somewhat difficult to understand how two massive clusters have built up to be of comparable mass and richness within such different environments. The only explanation we see is that Ophiuchus has built up long ago, as confirmed by its relaxed state (see paper I), while Coma is still in the process of forming, as illustrated by the series of papers by Adami et al. (see e.g. Adami et al. 2007). It would be interesting to test this hypothesis by comparing the degree of relaxation and the large-scale galaxy distribution for a large sample of rich clusters.

Acknowledgements. We thank the referee for her/his very useful comments. We acknowledge enlightening discussions with Emmanuel Bertin. We are extremely grateful to Andrea Biviano for giving us, and adapting for us, his program to fit the galaxy luminosity functions, for pointing out the Yasuda et al. (2001) paper, and for helpful discussions. We thank Florian Sarron for his help in extracting the individual extinctions for all the galaxies and Vincent Caillé for help measuring some of the galaxy magnitudes. F. D. acknowledges long-term support from CNES. J. M. O. M. M. M. was supported by the Brazilian agency CAPES ("Science without Borders" program 88888.789740/2014-00). This work is based on data obtained with MegaPrime/MegaCam (proposal 10AF02), a joint project of CFHT and CEA/DAPNIA, at the Canada-France-Hawaii Telescope (CFHT) which is operated by the National Research Council (NRC) of Canada, the Institut National des Sciences de l'Univers of the Centre National de la Recherche Scientifique of France, and the University of Hawaii. This research has made use of the NASA/IPAC Extragalactic Database (NED) which is operated by the Jet Propulsion Laboratory, California Institute of Technology, under contract with the National Aeronautics and Space Administration.

\section{References}

Adami, C., Durret, F., Mazure, A., et al. 2007, A\&A, 462, 411

Adami, C., Le Brun, V., Biviano, A., et al. 2009, A\&A, 507, 1225

Bertin, E., \& Arnouts, S. 1996, A\&AS, 117, 393

Biviano A., Durret, F., Gerbal, D., et al. 1996, A\&A, 311, 95

Cameron, L. M. 1990, A\&A, 233, 16

Durret, F., Laganá, T. F., Adami, C., \& Bertin, E. 2010, A\&A, 517, A94

Durret, F., Laganá, T. F., \& Haider, M. 2011, A\&A, 529, A38

Durret, F., Perrot, C., Lima Neto, G. B., et al. 2013, A\&A, 560, A78

Durret, F., Wakamatsu, K., Nagayama, T., Adami, C., \& Biviano, A. 2015, A\&A 583, A 124

Fukugita M., Shimasaku, K., \& Ichikawa, T. 1995, PASP, 107, 945

Johnston, M. D., Bradt, H. V., Doxsey, R. E., et al. 1981, ApJ, 245, 799

Mamon, G. A. 1992, ApJ, 401, L3

Martinet, N., Durret, F., Guennou, L., et al. 2015, A\&A, 575, A116

McCracken, H. J., Radovich, M., Bertin, E., et al. 2003, A\&A, 410, 17

Press, W. H., \& Schechter, P. 1974, ApJ, 187, 425

Schlafly, E. F., \& Finkbeiner, D. P. 2011, ApJ, 737, 103

Schlegel, D. J., Finkbeiner, D. P., \& Davis, M. 1998, ApJ, 500, 525

Silverman, B. W. 1986, Density Estimation for Statistics and Data Analysis (London : Chapman \& Hall)

Tully, R. B., \& Fisher, J. R. 1987, Nearby Galaxy Atlas (Cambridge: University Press)

Wakamatsu, K., \& Malkan, M. A. 1981, PASJ, 33, 57

Wakamatsu K., Malkan, M. A., Nishida, M. T., et al. 2005, Nearby Large-Scale Structures and the Zone of Avoidance, eds. K. P. Fairall, \& P. A. Woudt, ASP Conf Ser, 329189

Watanabe, M., Yamashita, K., Furuzawa, A., Kunieda, H., \& Tawara, Y. 2001, PASJ, 53, 605

Werner N., Zhuravleva, I., Canning, R. E. A., et al. 2016, MNRAS, 460, 2752

Yasuda, N., Fukugita, M., Narayanan, V. K., et al. 2001, AJ, 122, 1104 


\section{Appendix A: Typical errors on the magnitudes}

Table A.1. Typical errors on the measured magnitudes depending on the magnitude range.

\begin{tabular}{cc}
\hline \hline Magnitude range & Error on magnitude \\
\hline$r^{\prime}<20.0$ & 0.05 \\
$20.0<r^{\prime}<21.0$ & 0.10 \\
$21.0<r^{\prime}<22.0$ & 0.20 \\
$22.0<r^{\prime}<23.0$ & 0.5 \\
$23.0<r^{\prime}$ & 0.8 \\
\hline
\end{tabular}

The typical errors on the measured magnitudes are given in Table A.1 for various magnitude ranges.

\section{Appendix B: The first ten lines of the photometric catalogue}

The first ten lines of the photometric catalogue are shown in Table B.1.

Table B.1. First ten lines of the photometric catalogue of Ophiuchus, which includes 2818 galaxies and is available in ViZieR at the CDS.

\begin{tabular}{rrrrrrrrrr}
\hline \hline Name & $\begin{array}{r}\text { RA } \\
(J 2000.0)\end{array}$ & $\begin{array}{r}\text { Dec } \\
(\mathrm{J} 2000.0)\end{array}$ & $r^{\prime}$ & $r_{c t}^{\prime}$ & $r_{\text {var }}^{\prime}$ & $a$ & $b$ & PA & err PA \\
\hline OPHJ171014.79-233403 & 257.56162 & -23.56767 & 15.655 & 14.298 & 14.558 & 75.2 & 18.0 & 168.3 & 0.1 \\
OPHJ171014.90-232009 & 257.56207 & -23.33589 & 17.686 & 16.329 & 16.473 & 15.9 & 11.9 & 122.2 & 3.0 \\
OPHJ171014.98-234752 & 257.56241 & -23.79794 & 20.455 & 19.098 & 19.103 & 6.4 & 3.6 & 31.1 & 0.1 \\
OPHJ171014.99-234735 & 257.56244 & -23.79315 & 21.253 & 19.896 & 19.888 & 4.4 & 4.2 & 60.4 & 0.6 \\
OPHJ171015.56-225946 & 257.56485 & -22.99636 & 21.844 & 20.487 & 20.720 & 4.6 & 2.3 & 93.6 & 0.1 \\
OPHJ171016.02-232204 & 257.56674 & -23.36787 & 20.407 & 19.050 & 19.300 & 4.9 & 3.5 & 159.2 & 0.1 \\
OPHJ171016.06-232500 & 257.56693 & -23.41680 & 22.074 & 20.717 & 21.049 & 3.7 & 3.1 & 25.3 & 0.2 \\
OPHJ171016.08-230323 & 257.56699 & -23.05655 & 21.401 & 20.045 & 20.266 & 5.1 & 3.4 & 53.0 & 0.8 \\
OPHJ171016.27-232231 & 257.56781 & -23.37537 & 20.211 & 18.854 & 19.150 & 4.9 & 3.5 & 162.1 & 0.6 \\
OPHJ171016.27-233547 & 257.56781 & -23.59645 & 20.141 & 18.784 & 18.992 & 5.5 & 3.8 & 110.1 & 0.1 \\
\hline
\end{tabular}

Notes. The columns are: (1) name, (2) RA (2000.0), (3) Dec (J2000.0), (4) measured $r^{\prime}$ band magnitude with no extinction correction, (5) and (6) magnitudes $r_{c t}^{\prime}$ corrected for a constant value of $1.357 \mathrm{mag}$, and $r_{v a r}^{\prime}$ corrected for the individual extinction correction, (7) and (8) major axis $a$ and minor axis $b$ in arcseconds (the error bars on these two quantities are typically 0.1 arcsec, so they are not given for every galaxy), (9) position angle $P A$ of the major axis, (10) error on the position angle. We do not give individual errors on the observed magnitudes, but their typical values are given in Table A.1. 